\title{
Integrative Solutions in Online Crowdsourcing Innovation Challenges
}

\author{
Michael A. Zaggl \\ Aarhus University \\ zaggl@mgmt.au.dk
}

\author{
Yao Sun \\ University of South Florida \\ yaosun@usf.edu \\ Arvind Malhotra \\ University of North Carolina \\ arvind_malhotra@kenan- \\ flagler.unc.edu
}

\author{
Ann Majchrzak \\ University of Southern \\ California \\ majchrza@usc.edu
}

\begin{abstract}
Online crowdsourcing challenges are widely used for problem-solving and innovation. Existing theory has characterized such challenges as tools for tapping distant knowledge. By building on information processing theory we move beyond this characterization and present a perspective that describes innovation challenges as virtual places in which ideas are not simply submitted or commented upon but knowledge is integrated. This perspective shifts the role of crowdsourcing challenges from mere tools for gathering ideas to representing the locus of innovation. Our perspective suggests that three types of knowledge affect the quality of integrative solutions: elementary ideas, facts, and analogical examples. Based on a large dataset, we find that elementary ideas and analogical examples are related to increased solution quality, while facts are related to decreased solution quality. We expand the research on online crowdsourcing innovation challenges to include how crowd participants influence the quality of solutions through the content of their postings.
\end{abstract}

\section{Introduction}

Online crowdsourcing challenges are popular technologies for generating knowledge and innovations for knowledge seekers [1]. Examples range from question-and-answer communities [43,72] and online communities $[7,69]$ to lead user platforms $[37,71]$ and innovation challenges $[29,44]$. The information systems and innovation management literature has characterized crowdsourcing challenges as tools for gathering knowledge that is distant from the knowledge seeker and thus contains a high degree of novelty $[1,30,34,42,47,52]$. As tools for collecting ideas from distant knowledge, the ideas offered are generated outside of the challenge autonomously from others in the challenge [10,12,22,29,30,37,62,72].
An alternative approach in online crowdsourcing challenges is to ask participants to offer integrative solutions. Integrative solutions are proposed ideas that integrate knowledge shared by others [2]. They build on and integrate content posted earlier. The integration of prior knowledge has been researched in face-to-face working teams across many different contexts $[25,32,66]$. This research shows that integrative solutions are of higher quality than autonomously and individually created ideas [3,32], where quality is defined as more novel and containing a competitive advantage for the knowledge seeker.

Integrative solutions (instead of simple ad-hoc ideas) are particularly crucial when problems are illstructured, that is when a problem's "structure lacks definition" [60:181]. Ill-structured problems are opposed to well-structured problems, which often can be solved mathematically. By contrast, solving illstructured problems requires systemic and crossdisciplinary contributions. Multiple perspectives need to be taken into account, which can be accomplished by integrative solutions. Knowledge seekers increasingly use online crowdsourcing challenges for ill-structured problems. Examples are open strategy formulation challenges for particular organizations [46,70], product design or marketing challenges for specific brands $[12,23]$, and governmental or non-governmental organizations solving societal problems such as "grand challenges" [8,11]. Therefore, when crowds are involved in solving ill-structured problems, the development of high-quality integrative solutions is particularly sought. Integrative solutions also provide the advantage of consolidating ideas already produced by the crowds, thereby reducing the number of overall ideas having to be evaluated, and overall becoming more valuable for the knowledge seeker [52].

Of course, integrative solutions vary in their quality. Prior research indicates that the content of knowledge posted by the crowd may improve the quality of 
solutions generated in innovation challenges $[9,22,43,44,56]$. Yet there is a lack of research on the specific kinds of knowledge affecting the development of high-quality integrative solutions in online crowdsourcing challenges. Therefore, we ask the research question: Does the content of knowledge contributions in an innovation challenge affect the quality of integrative solutions?

Based on information processing theory $[15,48]$, we identify three types of knowledge that are likely to be posted by innovation challenge participants and which may be related to the generation of high-quality integrative solutions: (1) content containing nonintegrative, ad-hoc ideas that participants suggest to solve the innovation challenge question ("elementary ideas”), (2) content conveying participants' knowledge about details of the question ("facts"), and (3) content containing analogical examples known to a participant which have worked to address similar questions in different contexts (“analogical examples”). We develop hypotheses on how the quality of integrative solutions will benefit from prior posts offering (1) more elementary ideas, (2) fewer facts, and (3) more analogical examples. These hypotheses may appear counter-intuitive against the traditional team problemsolving literature [51,63], which suggests the limited value of idea brainstorming, assumes a positive value of sharing facts, and decries analogies. Based on information processing theory, we argue that in online innovation challenges - where elaborate back-and-forth conversations are limited-information that is most easily processed will foster higher quality integrative solutions. Facts are difficult to process because, in the context of a crowd, they are not easily verifiable or refutable without significant extra work, discouraging the creation of high-quality integrative solutions. In contrast, elementary ideas and analogical examples are more easily processable since they do not need to be verified nor particularly well-understood if their value is to stimulate new solution-ideas [15].

We empirically test our hypotheses on a dataset of more than three thousand posts offered in 21 innovation challenges calling for solutions for ill-structured problems. This dataset is special in the regard that crowd participants were encouraged to share any knowledge about the problem they would like, not just the ideas for solving the problem or comments on others' ideas (Majchrzak and Malhotra 2020). We coded the content of the posts for the three types of knowledge. Participants self-identified when a proposed solution was integrative by referencing prior posts. Integrative solutions were then judged for their usefulness and novelty to the organization. Our empirical tests support the hypotheses.
This paper contributes by extending the innovation challenge and crowdsourcing literature $[1,9,23,29]$ by extending the distant knowledge perspective [1] with the integrative knowledge perspective. Thus, we characterize online crowdsourcing innovation challenges not just as connectors between knowledge seekers and knowledge providers Specifically, we provide a detailed insight into which knowledge fosters or inhibits integration.

\section{Theoretical background}

We tackle the topic of ideation and knowledge creation through integrative solutions by building on information processing theory. Information processing theory describes how new information is perceived, filtered, and combined with prior knowledge in order to make decisions or execute actions [35,54]. The theory has been used to explain decision-making, strategizing, and knowledge creation of individuals and organizations [24,48,55,57]. Information systems researchers have extended information processing theory by incorporating information technology in the processing of information [53,54] specifically for the purpose of new knowledge creation involving groups [38]. Prominent examples of information processing enabled or enhanced by information technology are knowledge repositories (e.g., Wikipedia) and open source software development [4,5,21,39,40,67]. In these examples, new knowledge is created through interactive processing of currently available knowledge by individuals. More recently, this perspective has been applied to crowdsourcing $[43,44]$.

From the perspective of information-processing theory, crowdsourcing challenges can be specified as “dynamic spaces of collaboration” [19:670], which collect, process, and develop new knowledge. Diverse and fluid individuals engage in the crowd to different degrees and intensity. They draw and add knowledge to the common space of collaboration. The knowledge available in the space determines what knowledge they draw on. This knowledge, in turn, shapes what knowledge they contribute. As such, there is a dynamic interaction between the individuals' knowledge and the common knowledge space leading to the development of integrative new knowledge. Thus, crowds can be seen as "anarchical organizations" [14] but with the feature of dynamically developing new knowledge.

The aspect of dynamically developing knowledge substantially differs from the more traditional perspective on crowdsourcing as a search for distant knowledge $[1,17,52]$. In these traditional perspectives, crowdsourcing challenges are mere collections of ideas [17]. The ideas are then selected by the knowledge seeker [52]. The distant search perspective does not 
consider possible feedback mechanisms between the knowledge sources and the knowledge collected in the crowdsourcing challenge. Knowledge is not emerging but as a linear output of the inputs.

To apply the information processing perspective on crowdsourcing challenges to the notion of integrative solutions, we need to specify the mechanism (i.e., recombination and synthesis) and its basis (i.e., different types of knowledge) for integrative solutions emerging.

The way in which outcomes (problem solutions) are achieved in crowdsourcing challenges as dynamic spaces of collaboration is the (re)combination and synthesis of preexisting knowledge to build new solutions $[2,33,38,59]$. The openness and the diversity of the challenges push the potential of creating new knowledge that can serve as preexisting knowledgeleading to the development of integrative solutions. Thus, the potential of a crowdsourcing challenge to develop integrative solutions lies in its ability to make crowd participants recombine their knowledge with knowledge present in the challenge (i.e., previous knowledge posted by others). Thus, the emergence of valuable new knowledge is not random but influenced by the available knowledge.

As a basis for recombination and synthesis, different types of knowledge are to be differentiated. Information processing theory distinguishes four aspects of a problem [15,26]: goals, assumptions, elements, and operators. In the context of crowdsourcing challenges, goals need to be specified exogenously by the problem statement or challenge question and are not part of the solution-finding dynamics. Assumptions are "givens" related to the problem. In crowdsourcing, they are spontaneous factual responses to the problem by participants. We refer to them as facts. The third kind of knowledge, elements, are defined as changeable pieces of the problem that solve one or multiple aspects of the problem; we refer to these as ad-hoc or elementary ideas. The fourth kind of knowledge, operators, describes ways in which solutions to the problem can be implemented. These knowledge types illustrate concrete changes that are made to a possible solution. In the context of crowdsourcing challenges, analogical examples correspond to this type of knowledge. Analogical examples describe parallels to the focal problem often containing a parallel solution.

In sum, our perspective builds on information processing theory $[15,38,54]$ and applies it to crowdsourcing challenges. From this perspective, we see crowdsourcing challenges as dynamic spaces in which crowd participants can process knowledge existing in the challenge (which has been posted by other crowd participants) and mix - integrate- it with their own knowledge. This integration occurs as a process of recombination and synthesis of the knowledge. Thereby, three types of knowledge are distinguished: elementary ideas, facts, and analogical examples. Next, we hypothesize on the relation between the types of knowledge and the quality of integrative solutions.

\section{Hypotheses development}

\subsection{The effect of elementary ideas}

First, we consider the effect of elementary ideas as a type of knowledge. The information processing perspective, in general, suggests a positive relationship between elementary ideas and integrative solutions. Elementary ideas provide raw knowledge material, representing the basic building blocks for recombination and improvement $[33,38,59]$. Elementary ideas are used as the basic ingredients for integrative solutions. Their essence represents a potential that can be distilled into high-quality integrated solutions.

Our information processing perspective on crowdsourcing challenges suggests a positive relationship between elementary ideas and integrative solutions not because the elementary ideas are precisely represented in the integrative solution proposed, but because prior elementary ideas may spark new possibilities for solving the problem when considered in the context of other knowledge offered [6]. On their own (i.e., when not processed) elementary ideas are not contributive to the problem-solving outcome. Their limited potential in isolation is supported by research on brainstorming, face-to-face as well as online, shows that mere collections of ideas without the attempt of integration yield very poor innovation outcomes $[50,51]$. They are insufficient in isolation but a crucial basis for integrative solutions. Only when integrating an elementary idea with other knowledge, aspects or even inferences from the idea may be sufficient to generate novel combinations in one's creative mind [20].

Besides their positive influence, elementary ideas lack a detrimental effect even if they are not helpful to the problem at hand. Elementary ideas do not disturb the information processing process even if they are not consistent with others' knowledge and cannot be processed [15]. As long as the goal of the innovation challenge is sufficiently clear, elementary ideas can be unclear or even straight out wrong. Others do not need to understand them completely to be able to decide whether they are useful or it is impossible to build on them [15]. Thus, elementary ideas do no harm information processing even if they are useless. The positive potential and the lack of a disturbing influence leads us to our first hypothesis. 
Hypothesis 1 (H1): There is a positive relationship between the number of elementary ideas shared by the crowd and the quality of integrative solutions.

\subsection{The effect of facts}

Facts are assumptions about how the world operates with respect to the problem [15]. They may describe the severity or the frequency of the problem, documented customer needs to be considered during the solving of the problem, or reference to sources about possible causes of the problem.

Our perspective of information processing theory predicts a negative relationship between facts and the quality of integrative solutions in online crowdsourcing innovation challenges. Factual assumptions require deeper processing than elementary ideas because assumptions are shaped by the individual participant's mental model about the problem as well as the individual's background and experiences, both are not explicit to others [15]. Thus, integrating facts into solutions becomes difficult in the context of crowdsourcing. Particularly when it comes to illstructured problems, facts are problematic to process because the relevant assumptions to bring to bear on the problem are not always clear at the outset of problemsolving. From research in contexts other than crowdsourcing, the difficulty of processing factual assumptions has been shown to harm performance [16], get confused with unrelated knowledge when not clearly articulated [51], and harm the reuse since assumptions thought to be important for reuse may not be relevant [64]. Facts can contradict other facts, making it difficult to distinguish useful knowledge content from useless ones in order to better understand the problem [49,61]. In crowdsourcing challenges, such contradictions can develop into unproductive discussions about the correctness of one fact over the other, distracting from the goal of solving the problem. Thus, facts are likely to add unnecessary "noise", which is in the best case useless and in the worst case so difficult to process that they confuse and distract the crowds. Thus, we put forward the following hypothesis.

Hypothesis 2 (H2): There is a negative relationship between the number of facts shared by the crowd and the quality of integrative solutions.

\subsection{The effect of analogical examples}

Finally, we consider the effect of analogical examples on the quality of integrative solutions. Analogical examples, as defined in the framework as parallel (partial) parallel solutions to the focal problem, represent solutions for problems that have parallels to the focal problem. Analogical examples can help to solve the problem by describing personal experiences with solutions that have worked in other contexts [18].

Information processing theory ascribes a powerful role to analogical examples, especially when it comes to solving problems demanding high levels of creativity. Symbolically speaking, analogical examples provide bridges between the focal problem and a solution that works (partly) for another problem. The more remote the analogical (part-)solution is from the focal problem (i.e., the longer the bridge), the higher the creative potential for developing a novel and working solution [13].

Similar to our information processing perspective, the traditional perspective on online crowdsourcing recognizes the role of analogical examples. The notion of distant knowledge in the traditional perspective [2] is essentially a form of describing analogies. However, this view does not consider the need to synergizing the content of analogical examples but the information processing perspective advocates the need for synergizing. As such, analogical examples are not helpful for the focal problem since they explicitly relate to the analogical, remote, problem. Thus, integration is necessary. Prior research supports this by arguing that analogical examples are much more effectively processed by groups than by individuals [30]. Analogical examples are easy to be processed. They allow crowd participants to take others' perspectives without the need to know them [1,22,75]. Although analogical examples are not necessarily meaningful to all crowd participants, they only need to be sufficiently recognized to trigger a memory that may help to create a new solution. Further, analogical examples render a deep involvement in the context of crowd participants obsolete. Crowd participants can build on analogical examples without being deeply invested. Thus, we put forward our third hypothesis.

Hypothesis 3 (H3): There is a positive relationship between the number of analogical examples shared by the crowd and the quality of integrative solutions.

\section{Research method}

To test our hypotheses, we used a dataset that allowed participants to share knowledge about the problem without limitations to constrain the knowledge sharing or the feedback on others' suggestions. As such, this dataset allowed us to test our hypotheses on integration and the types of knowledge shared prior to the integrative solution being offered. Additionally, the dataset asked crowd participants to solve ill-structured problems; as such, integrative solutions were more important than might be the case if the problems to solve were more structured. Twenty-one crowdsourcing 
innovation challenges were included in this unique dataset.

The manner in which the data were obtained is described in [45] the data are open to the public for analysis. The 21 challenges were created with a sponsoring organization following specific protocols for generalizability across the challenges. Each of the innovation challenges (a) posed a clearly formulated illstructured problem, (b) broadcasted the open call to participate in the event to a set of multiple mailing lists specifically relevant to the call to attract a diverse set of crowd participants, (c) used its own virtual workspace platform to run the event which was standardized across the challenges by using the same platform provider (Brightideas.com), (d) ran the innovation challenge for similar durations (7 to 10 days), and (e) offered similar small incentives for the top 3 most novel solutions as determined by the sponsoring organization. In total, the dataset contains 3171 posts from 486 unique users, who had posted at least once.

Publicly known companies or governmental agencies who sought solutions to strategic problems sponsored these innovation challenges. Each challenge assembled a unique crowd. The sponsoring organizations were identified based on universityaffiliated partnerships, in which the Chief Innovation Officer or the Chief Executive Officer/Director to implement our challenges. The crowds were asked to respond to a general strategic question about suggestions for the companies' future development plans. Invitations were broadcast on multiple mailing lists corresponding to each organization's activities to recruit appropriate participants (e.g., for a toy company, the call was posted on a mailing list that young mothers are actively involved).

In all the challenges, the participants were asked to follow the same process. The participants were required first to register and then chose a pseudonym. The home page described the challenge problem/question and encouraged participants to post discussion thread starters, post comments on discussion threads started by others, and vote on other's posts with an up-or-down vote. When participants logged in, they saw the five most recent posts.

All posts were structured as discussion threads similar to crowd-based innovation challenges/online communities and all participants were required to register before posting content [72]. To ensure anonymity, participants were also asked to select a user name other than their real names to be displayed when posting. As the users logged in, they were presented with a home page describing the challenge and encouraging them to share their knowledge about the challenge. Following the welcoming sentences, the most recent threads were shown which could be clicked to reveal the full contents. On each of the thread's pages, participants could post their comments on the previous content; if participants found content that needed supplementing, they could also initiate a new discussion thread.

\subsection{Categorization of knowledge types}

Our framework hypothesizes that integrative solutions are related to certain knowledge types previously posted in the crowdsourcing challenge. To identify these knowledge types, we categorized all posts. We followed the knowledge management literature $[23,31,44,71]$ to break down the knowledge content of the postings into elementary ideas, facts, analogical examples (to construct our independent variables), integrative solutions (to construct our dependent variable), paradoxes (a control variable that is necessary given earlier research $[43,44])$, and posts containing no knowledge.

The categorization was conducted by two coders after all the challenges were closed. The two coders are experts in innovation management. One of the coders is an author of this paper and the other one a business analyst hired for the categorization tasks. In a first step, the coders independently read all 3171 posts and then decided for each post whether it contains any relevant knowledge at all. Posts containing no knowledge typically only said "Yes, I agree" or "Not so sure about this". These posts were labeled as "Others". Each of the remaining posts was coded in accordance with the knowledge content types based on the coders' review of both the title and the main body of each post. A reasonable inter-coder reliability (Cohen's Kappa = $0.74, \mathrm{p}<.001$ ) was obtained at the completion of the coding process [13], and disagreements were resolved through subsequent collaborative reading and coding. As a result, a total of 213 integrative solutions were coded in addition to the other knowledge types of posts. The resulting categorization was used to construct the variables, which we describe in the following.

\subsection{Variable construction}

As our independent variables, we counted the number of each knowledge content type that occurred in a window of five posts prior to each integrative solutions. For each hypothesis, a variable was constructed, which resulted in the variables: \#Facts in window, \#Elementary ideas in window, \#Analogical examples in window.

To construct our dependent variable-integrative solution quality - we followed the following procedure. First, we asked senior executives (either chief innovation officer or for smaller firms a c-suit 
executive) in the organizations that sponsored the challenged to rate ideas from their respective crowds on two dimensions. The first dimension was the novelty of the solution, i.e. the solution was one that the organization had previously been aware of or had contemplated themselves. The second dimension was the competitive advantage creating potential of the solution, i.e. if the solution was implemented it would give the organization a distinct advantage over the competition. Both dimensions were measured using a seven-point Likert scale.

Second, in order to normalize the ratings across the different challenges, since each executive only rated the solutions from their sponsor challenge, we used the procedure outlined in [36]. Using the ratings of the executives as subject matter expert ratings, we trained two independent raters to evaluate the integrative solutions across all the challenges. In addition, the raters were engaged in a thorough examination of the current offerings and strategies for each of the organizations and those of competitors. To accomplish this background training the raters used each organization's website as well as The Gale Business Insights Complete Collection of Business and Company Resources. The training and background research helped the independent raters develop repeatable heuristics to replicate the logic of senior executives ratings, and so to be able to rate all solutions across all the challenges vis-vis executive raters who only rated solutions rated to their organization's challenge. At the end of this procedure, the two independent raters' ratings demonstrated an interrater reliability of $71.2 \%$. Finally, the integrative solution quality score was calculated by combining the two dimensions of novelty and competitive advantage potential to create 3 levels of quality. Where 3 denoted when a solution had a high level of both dimensions (occurred 46 times), 2 was when the solution was high on one or the other dimension only (occurred 93 times), and a solution was rated as 1 (low) if it was low across both the dimensions (occurred 74 times).

We controlled for four other possibly confounding effects. First, we considered the posting position as the position of each integrative solution relative to the total length of the challenge. Later integrative solutions might be of lower quality because the crowd might have exhausted its creative potential. Alternatively, they could be of higher quality because more knowledge has been accumulated. Second, we take into account the number of different participants in the window (\#Different contributors in window). This variable controls whether the contributions stem from the same individuals. Third, we controlled for the prior number of integrative solutions in the window (\#Integrative Solutions in window). These prior integrative solutions could have captured the contributed knowledge. They also can be used as knowledge sources for developing integrative solutions. Fourth, we control for the knowledge content type of paradoxes in the window (\#Paradoxes in window). Paradoxes have been shown to influence ideas. Thus, it is possible that they are also used for integration and affect the quality of integrative solutions. Finally, for challenge-specific characteristics, we controlled by adding fixed effects on the challenge level.

\subsection{Hypotheses tests}

Table 1: Main results

\begin{tabular}{|c|c|c|}
\hline & \multicolumn{2}{|c|}{$\begin{array}{c}\text { Dependent variable: } \\
\text { Integrative Solution Quality }\end{array}$} \\
\hline & (1) & (2) \\
\hline \multirow[t]{2}{*}{ Posting position } & $-0.292^{*}$ & $-0.208^{* * *}$ \\
\hline & $(0.138)$ & $(0.057)$ \\
\hline \multirow{2}{*}{$\begin{array}{l}\text { \#Different } \\
\text { contributors } \\
\text { window }\end{array}$} & $=054$ & -0.053 \\
\hline & $(0.052)$ & $(0.028)$ \\
\hline \multirow[t]{2}{*}{$\begin{array}{l}\text { \#Integrative Solutions } \\
\text { in window }\end{array}$} & $-0.036^{* * *}$ & -0.028 \\
\hline & $(0.008)$ & $(0.015)$ \\
\hline \multirow[t]{2}{*}{$\begin{array}{l}\text { \#Paradoxes } \\
\text { window }\end{array}$} & 0.023 & 0.029 \\
\hline & $(0.041)$ & $(0.027)$ \\
\hline \multirow[t]{2}{*}{$\begin{array}{l}\text { \#Elementary ideas in } \\
\text { window (H1) }\end{array}$} & & $0.080^{* *}$ \\
\hline & & $(0.027)$ \\
\hline \multirow[t]{2}{*}{$\begin{array}{l}\text { \#Facts in window } \\
\text { (H2) }\end{array}$} & & $-0.219^{* * *}$ \\
\hline & & $(0.065)$ \\
\hline \multirow{2}{*}{$\begin{array}{l}\text { \#Anagogical } \\
\text { examples in window } \\
\text { (H3) }\end{array}$} & & $0.166^{* * *}$ \\
\hline & & $(0.022)$ \\
\hline Observations & 213 & 213 \\
\hline Adjusted $\mathrm{R}^{2}$ & 0.057 & 0.134 \\
\hline F Statistic & $\begin{aligned} & 1.852^{*}(\mathrm{df} \\
&=15 ; 197) \\
&\end{aligned}$ & 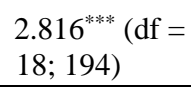 \\
\hline Note. & ${ }^{*} \mathrm{p}<0.05$ & $.01 ;^{* * *} \mathrm{p}<0.001$ \\
\hline
\end{tabular}

In order to account for unobserved heterogeneity on the level of the innovation challenges, we used fixedeffects regression models and clustered the standard errors. Table 1 shows the results. Model 1 uses the control variables only and Model 2 adds the independent variables.

The results show that Hypothesis 1 is supported. The number of elementary ideas in the window (\#Elementary ideas in window) is positively related to 
the quality of integrative solutions. Hypothesis 2 predicted that the number of facts in window (\#Facts in window) reduces the quality of integrated solutions. Our tests support this hypothesis by showing a negative significant effect. Finally, Hypothesis 3, which predicted a positive effect by the number of examples in the window (\#Examples in window) on the quality of integrative solutions is supported. Thus, all hypotheses of our framework are supported.

When comparing the magnitude of the effects, we find that the negative effect by facts is strongest $(-0.219$, $\mathrm{p}<.001)$, the examples have the second strongest effect (0.166, $\mathrm{p}<.001)$, and the smallest effect have elementary ideas $(0.080, \mathrm{p}<.01)$.

\section{Discussion}

In this study, we developed an information processing perspective on integrative solutions in crowdsourcing challenges. Our tests support our hypotheses. They show that facts (i.e., objective or perceived data) reduce the quality of following integrative solutions (H2) while elementary ideas and analogical examples increase the quality (H1 and H3).

\subsection{Implications to theory}

Our main contribution to the crowdsourcing challenge literature is to provide the notion of information process and integration as a new perspective of online crowdsourcing innovation challenges based on information processing. This perspective characterizes online crowdsourcing innovation challenges as tools to generate new knowledge rather than only to collect existing knowledge that is distant to the knowledge seeker. Our perspective regards crowdsourcing challenges as entities in which information processing can take place. Doing so, we extend and complement the currently prevailing perspectives on crowdsourcing innovation challenges, that is, the perspective of distant search for knowledge $[1,17,52]$. The perspective of distant search has focused on the generating of large numbers of elementary ideas or the quality of single isolated ideas as the main purpose of crowdsourcing challenges $[12,23,65]$. Specific emphasis has been put on the ability to tap "distant knowledge", which is knowledge that is remote and novel to the knowledge seeker [1,52]. By contrast, the notion of information processing to develop integrated solutions takes crowdsourcing one step further and looks at the crowds' ability to collaborate and integrate its own knowledge. It elevates the importance of crowdsourcing challenges as sociotechnical artifacts. Crowdsourcing challenges are not merely tools for collecting a large number of suggestions but virtual spaces for developing solutions.

Similarly, the notion of integrative solutions extends and complements the strong focus on the participants and their characteristics as a source of knowledge, which is a dominant perspective in research on crowdsourcing $[18,23,27,29,37,41,58,72]$ and related contexts such as open source software development $[28,68]$. Instead of providing just a tool to tap participants' knowledge, crowdsourcing challenges become the locus of innovation in which participants actively engage in order to develop integrative solutions. This put the participants into an informationprocessing role. Participants not only throw their private knowledge towards the knowledge seeker. Instead, they try to build on earlier elementary ideas and contextualize their contributions (as we have shown in $\mathrm{H} 1$ and H3). Facts as difficult to process knowledge inhibit novel and useful qualitative salutation to emerge. Thus, participants are not only the carriers of knowledge but also actively build upon each other's knowledge and generate emergent knowledge in the form of integrative solutions.

\subsection{Practical implications}

Our results have several implications for managers and designers of crowdsourcing challenges. We recommend stimulating the posting of elementary ideas and analogical examples. This can be achieved by triggering or rewarding these kinds of posting. For example, as soon as the discussion in the crowd becomes too abstract or detached from the context, the posting of analogical examples should be triggered by displaying stimuli such as "how could these ideas look like in the current situation met by our company?” Similarly, if too few elementary ideas are available to build on, a stimulus might trigger new ideas ("what possibilities, in general, could be interesting?”). In general, the posting of facts should be avoided by making the participants clear that demonstrating their knowledge is usually not helpful. In the near future, artificial intelligence could help to determine the timing for placing these triggers. The postings could be categorized automatically and predefined triggers could appear at the system's discretion.

Overall, knowledge seekers using crowdsourcing challenges ought to think of crowdsourcing as a dynamic and interacting locus of innovation instead of a mere technical tool to gather elementary ideas and then select the best fitting $[17,52]$. Innovation can take place inside the crowdsourcing challenge, which renders it the locus of innovation in which integrative solutions can emerge. 


\subsection{Limitations and future research}

In this study, we have adopted a knowledge sharing and integration view in the crowd based on information processing in order to focus on crowdsourcing as a locus of innovation. Thereby, we ignored differences in individuals, such as their creativity and extraversion. Although this is a useful step in order to establish our integrative perspective, further research is needed that considers both the information processing perspective and individual characteristic differences. It is likely that there is a correlation between the types of knowledge and the characteristics of the participants posting them. Perhaps, highly knowledgeable participants are more likely to post facts, more pragmatic or creative participants might tend to contribute elementary ideas or integrative solutions. Identifying these relationships between participant characteristics and the knowledge that they contribute helps to make crowdsourcing challenges more successful.

In this study, we were not able to study the specific contents of the knowledge contributions that are used for integrations and what contents are filtered. Are there some inherent characteristics of elementary ideas that make them conducive for being incorporated into integrative solutions? A more fine-grained categorization of the content posting could help to further increase our understanding of the innovation emerging. Relatedly, the mechanisms of selecting posted knowledge and using it for integration are completely unknown and require future research to take a cognitive stance. Laboratory settings that allow measuring the attention of participants (e.g., through eye-tracking) are needed to uncover those mechanisms.

Finally, our characterization renders crowdsourcing challenges as suitable for tackling ill-structured problems [60]. Future research should dig deeper into the relationship between the structuredness of problems and the information processing dynamics in the crowd. It is likely that different degrees of problem structuredness lead to different dynamics and influence the solution quality.

Altogether, we raise a call for future research on crowdsourcing challenges for innovation to emphasize the diversity of knowledge contributors, the heterogeneity of knowledge they contribute, and the mechanisms as well as the dynamics that connect posted knowledge to integrative solutions. Such research will lead to further design impactions encouraging the exchange and integration of diverse knowledge in online innovation crowdsourcing challenges.

\section{Conclusion}

As participants in crowdsourcing challenges make their diverse knowledge public, others can process this knowledge, build on it, and develop new integrative innovations. We shed new light with this information processing perspective on crowdsourcing challenges by investigating 21 crowdsourcing challenges and showing that the number of elementary ideas and the number of analogical examples most recently posted are positively related to the quality of the following integrative solutions. The number of posted facts, by contrast, is negatively related to the quality of integrative solutions. Our work extends the crowdsourcing challenge literature. Our notion shifts the locus of innovation to the dynamics of the text-based knowledge exchange in the crowdsourcing challenge.

\section{References}

[1] Afuah, A. and Tucci, C.L. Crowdsourcing as a Solution to Distant Search. Academy of Management Review, 37, 3 (2012), 355-375.

[2] Alavi, M. and Tiwana, A. Knowledge integration in virtual teams: The potential role of KMS. Journal of the American Society for Information Science and Technology, 53, 12 (2002), 1029-1037.

[3] Anderson, N., Potocnik, K., and Zhou, J. Innovation and Creativity in Organizations: A State-of-the-Science Review, Prospective Commentary, and Guiding Framework. Journal of Management, 40, 5 (March 2014), 1297-1333.

[4] Arazy, O., Daxenberger, J., Lifshitz-Assaf, H., Nov, O., and Gurevych, I. Turbulent Stability of Emergent Roles: The Dualistic Nature of Self-Organizing Knowledge Coproduction. Information Systems Research, 27, 4 (2016), 792-812.

[5] Arazy, O., Nov, O., Patterson, R., and Yeo, L. Information Quality in Wikipedia: The Effects of Group Composition and Task Conflict. Journal of Management Information Systems, 27, 4 (April 2011), 71-98.

[6] Baralou, E. and Tsoukas, H. How is New Organizational Knowledge Created in a Virtual Context? An Ethnographic Study. Organization Studies, 36, 5 (2015), 593-620.

[7] Barrett, M., Oborn, E., and Orlikowski, W. Creating Value in Online Communities: The Sociomaterial Configuring of Strategy, Platform, and Stakeholder Engagement. Information Systems Research, 27, 4 (2016), 704-723.

[8] Becker, J., Brocke, J. vom, Heddier, M., and Seidel, S. In Search of Information Systems (Grand) Challenges. Business \& Information Systems Engineering, 57, 6 (2015), 377-390.

[9] Blohm, I., Bretschneider, U., Leimeister, J.M., and Krcmar, H. Does collaboration among participants lead to better ideas in IT-based idea competitions? International Journal of Networking and Virtual Organisations, 9, 2 (2011), 106-122. 
[10] Boudreau, K.J. and Lakhani, K.R. Using the Crowd as an Innovation Partner. Harvard Business Review, 91, 4 (2013), 60-69.

[11] Chen, L., Baird, A., and Straub, D. Fostering Participant Health Knowledge and Attitudes: An Econometric Study of a Chronic Disease-Focused Online Health Community. Journal of Management Information Systems, 36, 1 (2019), 194-229.

[12] Chen, L., Marsden, J.R., and Zhang, Z. Theory and Analysis of Company-Sponsored Value Co-Creation. Journal of Management Information Systems, 29, 2 (2012), 141-172.

[13] Cohen, J. A coefficient of agreement for nominal scales. Educational and Psychological Measurement, 20, 1 (1960), 37-46.

[14] Cohen, M.D., March, J.G., and Olsen, J.P. A Garbage Can Model of Organizational Choice. Administrative Science Quarterly, 17, 1 (1972), 1-25.

[15] Cronin, M.A. and Weingart, L.R. Representational Gaps, Information Processing, and Conflict in Functionally Diverse Teams. Academy of Management Review, (2007).

[16] Daft, R.L. and Weick, K.E. Toward a Model of Organizations as Interpretation Systems. The Academy of Management Review, 9, 2 (1984), 284-295.

[17] Dahlander, L., Jeppesen, L.B., and Piezunka, H. How Organizations Manage Crowds: Define, Broadcast, Attract and Select. In M. Lounsbury, J. Sydow and H. Berends, eds., Research in the Sociology of Organizations. 2018.

[18] Ebner, W., Leimeister, J.M., and Krcmar, H. Community engineering for innovations: the ideas competition as a method to nurture a virtual community for innovations. R\&D Management, 39, 4 (2009), 342-356.

[19] Faraj, S., Krogh, G. von, Monteiro, E., and Lakhani, K.R. Special Section Introduction-Online Community as Space for Knowledge Flows. Information Systems Research, 27, 4 (2016), 668-684.

[20] Finke, R.A., Smith, S.M., and Ward, T.B. Creative Cognition: Theory, Research, and Applications. MIT Press, 1992.

[21] Forte, A., Larco, V., and Bruckman, A. Decentralization in Wikipedia Governance. Journal of Management Information Systems, 26, 1 (2009), 49-72.

[22] Füller, J., Hutter, K., and Faullant, R. Why Co-Creation Experience Matters? Creative Experience and Its Impact on the Quantity and Quality of Creative Contributions. $R \& D$ Management, 41, 3 (2011), 259-273.

[23] Füller, J., Hutter, K., Hautz, J., and Matzler, K. User Roles and Contributions in Innovation-Contest Communities. Journal of Management Information Systems, 31, 1 (2014), 273-308.

[24] Galbraith, J.R. Organization Design: An Information Processing View. Interfaces, 4, 3 (1974), 28-36.

[25] Hargadon, A.B. and Bechky, B.A. When Collections of Creatives Become Creative Collectives: A Field Study of Problem Solving at Work. Organization Science, 17, 4 (2006), 484-500.

[26] Hayes, J.R. and Simon, H.A. Understanding Written Problem Instructions. In L.W. Gregg, ed., Knowledge and Cognition. Lawrence Erlbaum, Hillsdale, NJ, 1974, pp. 167-200.

[27] Howe, J. The Rise of Crowdsourcing. Wired Magazine, (2006).

[28] Howison, J. and Crowston, K. Collaboration Through Open Superposition: A Theory of the Open Source Way. MIS Quarterly, 38, 1 (2014), 29-50.

[29] Hutter, K., Füller, J., Hautz, J., Bilgram, V., and Matzler, K. Machiavellianism or Morality: Which Behavior Pays Off In Online Innovation Contests? Journal of Management Information Systems, 32, 3 (2015), 197228.

[30] Jeppesen, L.B. and Lakhani, K.R. Marginality and Problem-Solving Effectiveness in Broadcast Search. Organization Science, 21, 5 (2010), 1016-1033.

[31] Kelley, T. and Kelley, D. Creative Confidence: Unleashing the Creative Potential Within Us All. Crown Business, New York, NY, 2013.

[32] Knippenberg, D. van. Team Innovation. Annual Review of Organizational Psychology and Organizational Behavior, 4, 1 (2017), 211-233.

[33] Kogut, B. and Zander, U. Knowledge of the Firm, Combinative Capabilities, and the Replication of Technology. Organization Science, 3, 3 (1992), 383-397.

[34] Koh, T.K. Adopting Seekers' Solution Exemplars in Crowdsourcing Ideation Contests: Antecedents and Consequences. Information Systems Research, (2019).

[35] Lachman, R., Lachman, J.L., and Butterfield, E.C. Cognitive Psychology and Information Processing: An Introduction. Psychology Press, 1979.

[36] Lamastra, C.R. Software innovativeness. A comparison between proprietary and Free/Open Source solutions offered by Italian SMEs. R\&D Management, 39, 2 (2009), 153-169.

[37] Leimeister, J.M., Huber, M., Bretschneider, U., and Krcmar, H. Leveraging Crowdsourcing: ActivationSupporting Components for IT-Based Ideas Competition. Journal of Management Information Systems, 26, 1 (2009), 197-224.

[38] Li, Y. and Kettinger, W.J. An Evolutionary InformationProcessing Theory of Knowledge Creation. Journal of the Association for Information Systems, 7, 1 (2006), 25.

[39] Lindberg, A., Berente, N., Gaskin, J., and Lyytinen, K. Coordinating Interdependencies in Online Communities: A Study of an Open Source Software Project. Information Systems Research, 27, 4 (2016), 751-772.

[40] Liu, J. and Ram, S. Who Does What: Collaboration Patterns in the Wikipedia and Their Impact on Article Quality. ACM Transactions on Management Information Systems, 2, 2 (2011), 1-23.

[41] Love, J. and Hirschheim, R. Crowdsourcing of Information Systems Research. European Journal of Information Systems, 26, 3 (017), 315-332.

[42] Majchrzak, A., Cherbakov, L., and Ives, B. Harnessing the Power of the Crowds with Corporate Social Networking Tools. MIS Quarterly Executive, 8, 2 (2009).

[43] Majchrzak, A., Malhotra, A., and Zaggl, M.A. How Open Crowds Self-Organize. Academy of Management Discoveries, forthcoming, (2020).

44. Majchrzak, A. and Malhotra, A. Effect of KnowledgeSharing Trajectories on Innovative Outcomes in 
Temporary Online Crowds. Information Systems Research, 27, 4 (2016), 685-703.

[45] Majchrzak, A. and Malhotra, A. Unleashing the Crowd: Collaborative Solutions for Wicked Business and Societal Problems. Springer International Publishing, 2020.

[46] Malhotra, A., Majchrzak, A., and Niemiec, R.M. Using Public Crowds for Open Strategy Formulation: Mitigating the Risks of Knowledge Gaps. Long Range Planning, 50, 3 (2017), 397-410.

[47] Mo, J., Sarkar, S., and Menon, S. Know When to Run: Recommendations in Crowdsourcing Contests. MIS Quarterly, 42, 3 (2018), 919-944.

[48] Newell, A. and Simon, H.A. Human Problem Solving. Longman Higher Education, 1972.

[49] Ohlsson, S. Towards Intelligent Tutoring Systems that Teach Knowledge Rather than Skills. In New Directions in Educational Technology. Springer Berlin Heidelberg, 1992, pp. 71-96.

[50] Paulus, P.B., Dzindolet, M., and Kohn, N.W. Collaborative Creativity-Group Creativity and Team Innovation. In Handbook of Organizational Creativity. Elsevier, 2012, pp. 327-357.

[51] Paulus, P.B. Fostering creativity in groups and teams. In J. Zhou and C.E. Shalley, eds., Handbook of Organizational Creativity. Taylor and Francis, 2008, pp. 165-188.

[52] Piezunka, H. and Dahlander, L. Distant Search, Narrow Attention: How Crowding Alters Organizations' Filtering of Suggestions in Crowdsourcing. Academy of Management Journal, 58, 3 (2015), 856-880.

[53] Proctor, R.W. and Vu, K.-P.L. The Cognitive Revolution at Age 50: Has the Promise of the Human InformationProcessing Approach Been Fulfilled? International Journal of Human-Computer Interaction, 21, 3 (2006), 253-284.

[54] Proctor, R.W. and Vu, K.-P.L. Cumulative Knowledge and Progress in Human Factors. Annual Review of Psychology, 61, 1 (2010), 623-651.

[55] Puranam, P., Alexy, O., and Reitzig, M. What's "New" about New Forms of Organizing? Academy of Management Review, 39, 2 (2014), 162-180.

[56] Riedl, C. and Woolley, A.W. Teams vs. Crowds: A Field Test of the Relative Contribution of Incentives, Member Ability, and Emergent Collaboration to Crowd-Based Problem Solving Performance. Academy of Management Discoveries, 3, 4 (December 2017), 382-403.

[57] Rogers, P.R., Miller, A., and Judge, W.Q. Using Information-Processing Theory to Understand Planning/Performance Relationships in the Context of Strategy. Strategic Management Journal, 20, 6 (1999), 567-577.
[58] Schlagwein, D. and Bjorn-Andersen, N. Organizational Learning with Crowdsourcing: The Revelatory Case of LEGO. Journal of the Association for Information Systems, 15, 11 (2014), 754-778.

[59] Seshadri, S. and Shapira, Z. The flow of ideas and timing of evaluation as determinants of knowledge creation. Industrial and Corporate Change, 12, 5 (2003), 10991124.

[60] Simon, H.A. The Structure of Ill Structured Problems. Artificial Intelligence, 4, 3-4 (1973), 181-201.

[61] Streufert, S. and Nogami, G. Cognitive complexity and team decision making. In R.W. Swezey and E. Salas, eds., Teams: Their Training and Performance. Ablex Publishing, Westport, CT, 1992, pp. 127-151.

[62] Surowiecki, J. The Wisdom of Crowds. Anchor, 2005.

[63] Sutton, R.I. and Hargadon, A. Brainstorming Groups in Context: Effectiveness in a Product Design Firm. Administrative Science Quarterly, 41, 4 (1996), 685-718.

[64] Szulanski, G., Ringov, D., and Jensen, R.J. Overcoming Stickiness: How the Timing of Knowledge Transfer Methods Affects Transfer Difficulty. Organization Science, 27, 2 (2016), 304-322.

[65] Terwiesch, C. and Xu, Y. Innovation Contests, Open Innovation, and Multiagent Problem Solving. Management Science, 54, 9 (2008), 1529-1543.

[66] Tiwana, A. and McLean, E.R. Expertise Integration and Creativity in Information Systems Development. Journal of Management Information Systems, 22, 1 (2005), 1343.

[67] Tullio, D.D. and Staples, D.S. The Governance and Control of Open Source Software Projects. Journal of Management Information Systems, 30, 3 (2013), 49-80.

[68] von Krogh, G., Haefliger, S., Spaeth, S., and Wallin, M.W. Carrots and Rainbows: Motivation and Social Practice in Open Source Software Development. MIS Quarterly, 36, 2 (2012), 649-676.

[69] Wasko, M.M. and Faraj, S. Why Should I Share? MIS Quarterly, 29, 1 (2005), 35-57.

[70] Whittington, R., Cailluet, L., and Yakis-Douglas, B. Opening Strategy: Evolution of a Precarious Profession. British Journal of Management, 22, 3 (2011), 531-544.

[71] Yan, J. (Kevin), Leidner, D.E., and Benbya, H. Differential Innovativeness Outcomes of User and Employee Participation in an Online User Innovation Community. Journal of Management Information Systems, 35, 3 (2018), 900-933.

[72] Zhao, L., Detlor, B., and Connelly, C.E. Sharing Knowledge in Social Q\&A Sites: The Unintended Consequences of Extrinsic Motivation. Journal of Management Information Systems, 33, 1 (2016), 70-100. 